\title{
Do female rainbow kribs choose males on the basis of their apparent aggression and boldness? A non-correlational mate choice study
}

\author{
Ulrike Scherer $^{1}$ (D) Jean-Guy J. Godin ${ }^{2}$ (D) Wiebke Schuett ${ }^{1,3}$ (D) \\ Received: 17 May 2019 / Revised: 17 December 2019 / Accepted: 20 December 2019 / Published online: 3 March 2020 \\ (C) The Author(s) 2020
}

\begin{abstract}
Consistent between-individual differences in behaviour, known as personality differences, are heritable and have consequences for individual survival and reproductive success. Therefore, it is likely that personality differences are not just under natural but also under sexual selection. Indeed, the recently developed idea that individuals choose their mate based on its personality finds empirical support. However, most studies on mate choice based on personality traits are correlative pioneering work and there is a paucity of experimental studies that test for causality by disentangling personality measures from other, potentially correlated traits that may be important during mate choice. Here, we tested female preference for the apparent level and consistency of either male aggression (measured as mean distance of approach towards an animated opponent, manipulated by locating males at a fixed distance) or male boldness (measured as activity under a simulated predation threat, manipulated using a gradient in ambient water temperature) in a bi-parental West African cichlid, Pelvicachromis pulcher. Females could observe the apparent behaviour of paired stimulus males and were allowed to choose between the two stimulus males in a subsequent choice test. We found no direct effect of male apparent aggression/boldness on female choice, but an indirect effect such that female preference for the apparently bold male increased with increasing within-male pair contrast in their apparent level of boldness. Our results indicate females consider male boldness per se during mate choice, suggesting male boldness is sexually selected in our study species.
\end{abstract}

\section{Significance statement}

Ever since Darwin introduced the concept of sexual selection, female choice has been studied extensively. However, the hypothesis that consistent between-individual differences in behaviour (known as personality differences) affect mate choice is relatively new. Correlative studies support this idea but provide only suggestive evidence. Here, we used behavioural manipulations in order to disentangle male behaviour from other, potentially correlated male traits, allowing us to test for causality between female choice and personality differences in male aggression and boldness (both in level and consistency of behaviour) in a bi-parental cichlid. We found no overall female preference for male apparent behaviour, but female preference for the boldappearing male increased with increasing between-male contrast in apparent boldness. Our results indicate a causal link between female choice and male boldness. In future, behavioural manipulations using a temperature gradient could provide further valuable insights.

Keywords Cichlid $\cdot$ Behavioural manipulation $\cdot$ Sex difference $\cdot$ Mating preference $\cdot$ Pelvicachromis pulcher $\cdot$ Personality

Communicated by J. Frommen

Electronic supplementary material The online version of this article (https://doi.org/10.1007/s00265-019-2794-5) contains supplementary material, which is available to authorized users.

Ulrike Scherer

u.k.scherer@gmail.com

1 Institute of Zoology, Universität Hamburg, Martin-Luther-King Platz 3, 20146 Hamburg, Germany
2 Department of Biology, Carleton University, 1125 Colonel By Drive, Ottawa, ON, Canada

3 School of Life Sciences, University of Sussex, Falmer, Brighton BN1 9QG, UK 


\section{Introduction}

Consistent differences in individual behaviour, also known as personality differences, temperaments or coping styles (Gosling 2001; Réale et al. 2007), are moderately heritable (Ariyomo et al. 2013; Patrick et al. 2013) and have diverse fitness consequences (Dingemanse and Réale 2005; Smith and Blumstein 2008; Ariyomo and Watt 2012), including various effects on reproductive success (e.g. Spoon et al. 2006; Schuett et al. 2011a; Cain and Ketterson 2013; Laubu et al. 2016). Therefore, it seems likely that sexual selection may play a key role in shaping stable personality variation within populations (Schuett et al. 2010). Indeed, previous studies showed that personality traits, such as boldness (Godin and Dugatkin 1996; Scherer et al. 2017b), aggression (Ophir and Galef 2003; Kralj-Fišer et al. 2013) and exploratory behaviour (Schuett et al. 2011b; Pogány et al. 2018), affect mate choice in several species.

However, our understanding of how sexual selection relates to personality differences remains incomplete. The few empirical data collected so far differ regarding their direction of effects found. That is, previous studies on mate choice for aggressiveness and boldness have revealed either directional (Scherer et al. 2018a), assortative (KraljFišer et al. 2013; Pogány et al. 2018), dis-assortative (Scherer et al. 2017b) or no effects (Laubu et al. 2017; Scherer and Schuett 2018) of individual behavioural types on mating preferences. Moreover, existing studies have often focused on potential effects of the behavioural level of a given personality trait on female mating preferences while neglecting the importance of individual differences in the consistency of its expression (but see Scherer et al. 2017b, 2018a). Additionally, most previous studies on the potential role of individual personality on mate choice are correlative in nature (but see Schuett et al. 2011b). To test for causality, we need behavioural manipulations that allow us to decouple the personality trait of interest from other, correlated traits that may influence mate choice. For examples, in the guppy, Poecilia reticulata, body colouration and boldness are positively correlated with each other (Godin and Dugatkin 1996). Using a correlative mate choice set-up, one can therefore not conclusively distinguish between female preference for male colouration and male boldness (Godin and Dugatkin 1996). Only the manipulation of behaviour, disentangling the behavioural trait of interest from other traits being correlated to it, allows testing for causality (Godin and Dugatkin 1996; Schuett et al. 2011b). However, such experimental evidence for mating preferences for behavioural traits barely exists (Godin and Dugatkin 1996; Schuett et al. 2011b).

In two pioneering personality-mate choice studies, we tested female rainbow kribs (Pelvicachromis pulcher) for their mating preference in a correlative context. We assessed female preferences for the level and consistency of male aggression (Scherer et al. 2018a) and boldness (Scherer et al. 2017b). We found both these male personality traits to be linked with female choice. Regarding male aggression, females preferred consistent over inconsistent males, but the level of male aggression did not affect female preference (Scherer et al. 2018a). Further, females showed a dis-assortative preference for the level of male boldness and an assortative preference for the consistency of this behavioural trait (Scherer et al. 2017b). These two correlative studies provide only suggestive evidence for the direction of selection. In the current study, we aimed to test for a potential causal link between the aforementioned two male personality traits and the previously observed mating preference patterns in female rainbow kribs (Scherer et al. 2017b, 2018a). We experimentally manipulated both the level and consistency of aggression (experiment 1) and boldness (experiment 2) in male rainbow kribs and tested for an effect of these apparent traits on female mating preferences. In the first experiment, we manipulated the level and consistency of apparent male aggression (measured as average distance of approach towards an opponent) by placing a male in a clear Plexiglas cylinder located at a fixed distance from an animated same-sex conspecific opponent presented on a nearby computer screen. The average distance from such an animated opponent is a good proxy for aggressiveness in our model species (Scherer et al. 2017a). In the second experiment, we manipulated the level and consistency of apparent boldness (measured as activity under a simulated predation threat) using a gradient in ambient water temperature to manipulate the activity level of the focal male. In fishes, locomotor activity correlates positively with ambient water temperature (Castonguay and $\mathrm{Cyr}$ 1998; Forsatkar et al. 2016). In both experiments, females were allowed to choose between two stimulus males that were manipulated to differ in their apparent level or consistency of behaviour after prior observation of the apparent behaviour. Then, we tested female preference for the level and consistency of male behaviour by following up on two alternative hypotheses: (dis-)assortative mate choice vs. directional mate choice for or against a behavioural trait (Schuett et al. 2010). Testing female preference for male apparent behaviour relies on the assumption that fish can remember individual conspecifics and their behaviour. Fishes can identify and recognise con- and heterospecifics for several weeks (reviewed in Bshary et al. 2001). Further, fishes remember social information and subsequently use this information to make reproductive decisions (Schlupp et al. 1994; 
Doutrelant and McGregor 2000; Ophir and Galef 2003; Witte and Godin 2010).

\section{Methods}

\section{Test fish and holding conditions}

Test fish were sourced from the Universität Hamburg and local suppliers. All individuals were held in family (sibling) groups (total $=10$ families) under standardised holding conditions (100-200 1 tanks, $25 \pm 1{ }^{\circ} \mathrm{C}$, aerated and filtered water, weekly water changes). Fish were fed with Artemia spp. once daily on 5 days per week in their holding tanks. During experimentation, individuals were kept individually in smaller tanks $(25 \times 50 \times 25 \mathrm{~cm})$ and fed daily to maintain constant conditions between trials. The latter tanks were equipped with an immersion heater, an internal filter and half a clay pot $(4 \times$ $8 \times 8 \mathrm{~cm})$ as shelter. Fish were uniquely marked on their dorsal side with Visible Implant Elastomers (VIEs, VIE-Northwest Marine Technology, Shaw Island, WA, USA) of different colours for individual identification. VIEs do not affect mate choice in our study species (Schuett et al. 2017). Before experiments, all individuals were measured for their standard length using ImageJ (Schneider et al. 2012) (mean \pm SE standard lengths in experiment 1 : males $=4.69 \pm 0.02 \mathrm{~cm}$, females $=3.98 \pm 0.08 \mathrm{~cm}$; experiment $2:$ males $=5.38 \pm$ $0.04 \mathrm{~cm}$, females $=4.30 \pm 0.03 \mathrm{~cm}$ ). For all experimental trials, the water in test tanks was changed after every trial; water level was $10 \mathrm{~cm}$. Unless otherwise stated, the water temperature in experimental tanks was maintained at $25 \pm 1{ }^{\circ} \mathrm{C}$. During experimental trials, no humans were present in the experimental room to avoid disturbances and trials were video-recorded using an overhead video camera (Sony HDR-CX405). In both experiments, males were habituated to being in a clear Plexiglas cylinder $($ diameter $=8.0 \mathrm{~cm}$ ) twice for $10 \mathrm{~min}$, once on two consecutive days before the mate choice trials. Individuals became readily accustomed to these cylinders and did not show any behavioural signs of distress whilst in the cylinders. For all experiments, blinded methods were used. More specifically, all behavioural assessments were automated using a tracking software to minimise any observer bias. The experimenter was not aware of behavioural scores while conducting experimental trials.

\section{Experiment 1: female choice for male apparent aggression}

In experiment 1 (February-May 2017), we assessed female mating preference for the apparent level $(N=48$ preference assessments) and apparent consistency ( $N=48$ preference assessments) of male aggression (see "Mate choice trials" section). Before the mate choice trials, all males $(N=96)$ and females $(N=48)$ were tested for their natural aggressive behaviour twice with $48 \mathrm{~h}$ (range of $\pm 15 \mathrm{~min}$ ) between the two tests to determine the average level and inconsistency of behaviour (see "Aggression test"). We assessed personality differences using repeatabilities (for general principles, see e.g. Lessells and Boag 1987) and tested for sex differences in the level and inconsistency of behaviour (see Data analyses). Two days elapsed between the last aggression tests and the beginning of mate choice trials.

\section{Aggression test}

Male and female aggression was quantified separately and indirectly as the mean distance of approach $(\mathrm{cm})$ towards a computer-animated, same-sex conspecific opponent, as outlined in Scherer et al. (2017a). To begin an aggression test, we introduced two focal individuals, matched for sex and standard length, each into one of two adjacent test tanks (visually isolated from each other) that were aligned to face a computer monitor on their shorter axis (Fig. 1a, left panel, set-up with a grey background). After a 10-min acclimation period, during which the computer monitor was visually separated from test tanks, focal individuals were exposed to a computeranimated and unfamiliar same-sex, same-size opponent (to prepare the animations we used: $N_{\text {males }}=9, N_{\text {females }}=7$; size difference between the opponent and focal individuals $<3 \mathrm{~mm}$ ) for a test period of $11 \mathrm{~min}$. The simulated opponent was animated to swim back and forth horizontally along the width of a white computer screen (see Scherer et al. 2017a for details).

For all trials, the mean distance to the animated opponent was assessed for $10 \mathrm{~min}$ (we did not track the first minute of a video) using the tracking software Ethovision XT 11 (Noldus, Wageningen, The Netherlands). For each individual, we averaged the two mean distances to the opponent (obtained during the two aggression tests, see above) as a proxy measure of each fish's individual level of aggressiveness. Further, we assessed behavioural consistency of each individual as inconsistency: the absolute value of the difference between its mean distance of approach towards the animated opponent in the repeated aggression tests (Scherer and Schuett 2018; Scherer et al. 2017b, 2018a). Please note that large values indicate low consistency.

\section{Mate choice trials}

A mate choice trial consisted of an initial observation phase, followed immediately by a choice phase (Fig. 1). During the observation phase, a female was allowed to observe two stimulus males concurrently, with the males either differing in their apparent level of aggression (high vs. low) with consistency held constant (both fish consistent; Fig. 1a) or differing in their apparent consistency of aggression (consistent vs. inconsistent; Fig. 1b) with the average level of aggression held 

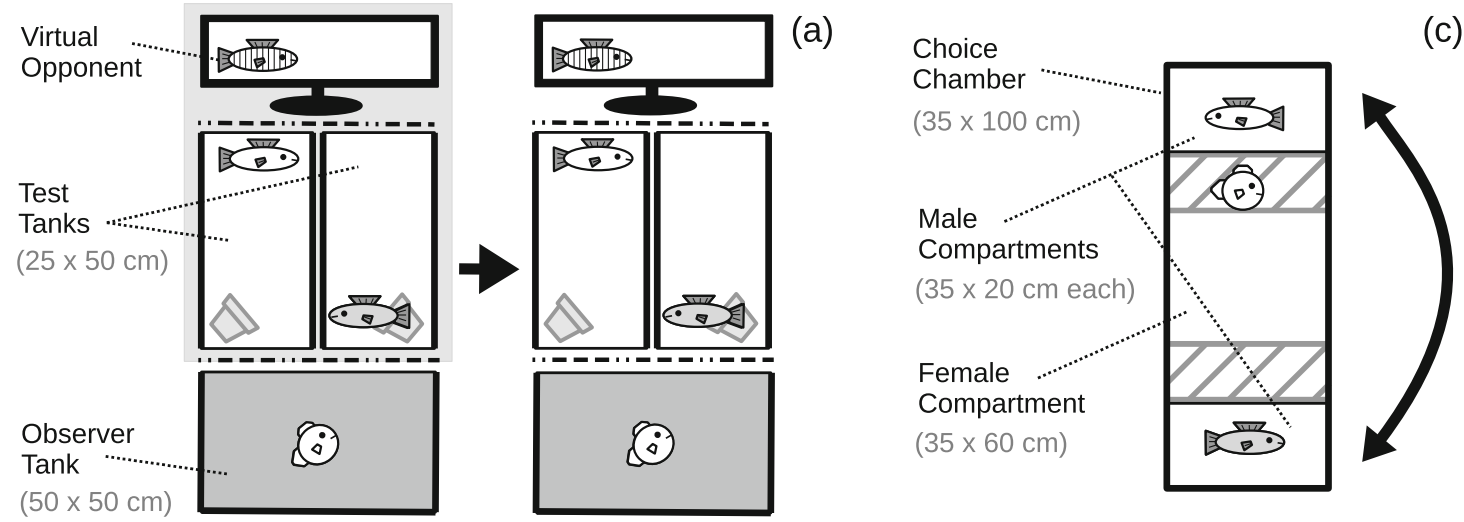

(c)

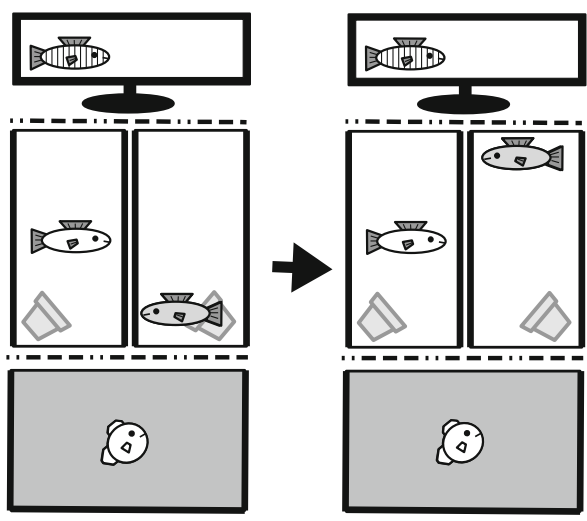

(b)

\section{LEGEND}

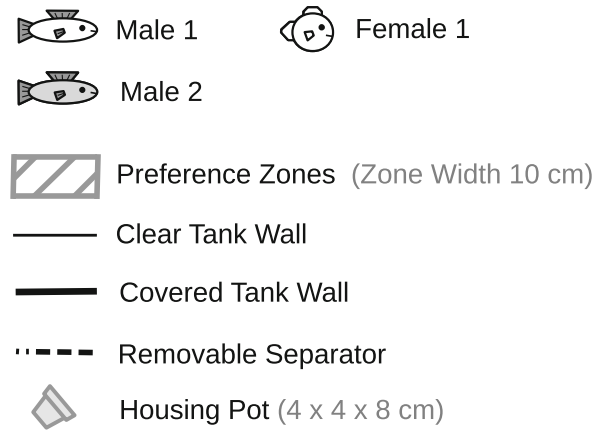

Fig. 1 Top-view schematic of the experimental set-up for the $\mathbf{a}, \mathbf{b}$ observation and subsequent $\mathbf{c}$ choice phase of mate choice trials. a The grey underlay (left panel) indicates the set-up for the aggression test. During two periods of the observation phase, we manipulated either a the level or b the consistency of male apparent aggression (mean distance towards an

animated opponent). Arrows indicate (a, b) the direction of manipulation between the first and second observation periods within a mate choice trial or (c) individual males that were switched in their position between recording periods. Grey tank background indicates black tank surrounding (including a black lid)

constant (intermediate aggression level for both males). Males were made to appear highly or less aggressive (or alternatively consistent or inconsistent) by manipulating their distance to an animated opponent moving on a computer monitor screen (Scherer et al. 2017a). The spatial position of each stimulus male was standardised by introducing them into separate clear Plexiglas cylinders $($ diameter $=8.0 \mathrm{~cm})$ that were placed on the bottom of their test tanks either close to $(4 \mathrm{~cm})$, intermediate to $(24 \mathrm{~cm})$ or far from $(44 \mathrm{~cm})$ the animated opponent so as to simulate high, intermediate or low aggression level in the stimulus male, respectively. Consistency was manipulated by changing (inconsistent aggression) or maintaining (consistent aggression) the distance to the animated opponent between two periods of the observation phase as follows.

Following $10 \mathrm{~min}$ of acclimatisation to the experimental tanks (Fig. 1a, b), we started the observation phase (22 min), which consisted of two consecutive 11-min periods. After the first observation period, we either changed or maintained the positions of the paired stimulus males according to their respective manipulation and allowed the fish to acclimatise for another $5 \mathrm{~min}$. When testing female preference for the apparent level of male aggression, both males differed in their apparent level of aggression but showed the same apparent behavioural consistency. During both observation periods, one of the paired stimulus males was placed in close proximity to the virtual opponent (apparent high-aggression male) and the other one further away from the opponent (the apparent low-aggression male) (Fig. 1a). Conversely, when testing female preference for consistency, we altered the position of one of the two stimulus males relative to the animated opponent between the two observation periods (thus simulating inconsistency in his aggression level), while keeping the position of the other male (thus simulating consistency in his aggression level) (Fig. 1b). We sham-changed the position of the consistent male, i.e. we moved the male but placed him back into his original position to control for potential effects of handling. We placed the apparently inconsistent male close to the opponent during one observation period and far from the opponent during the other observation period (in randomised order). The apparently consistent male was placed at an intermediate distance from the opponent during both observation periods (Fig. 1b). Thus, both stimulus males showed on average the same apparent level of aggression, but differed in their apparent behavioural consistency. Throughout the observation phase, the female was placed in 
a clear Plexiglas cylinder (diameter $20 \mathrm{~cm}$, placed in the centre of her tank) so that both males remained visible to her. During acclimatisation periods, removable opaque screens visually blocked the female observer tank, the stimulus males' tanks, and the computer monitor from each other.

We also carried out control mate choice trials (for both level and consistency of male aggression, respectively) in a similar manner to that described above, except that the computer screen monitor did not display a virtual conspecific opponent but only a static white background during the observation phase (Fig. 1a, b). Control trials were used to account for the possibility that differences in the manipulated distances between the observer female and the stimulus males per se could account for any subsequent female preference for either stimulus male. Hence, there were four different treatments for the observation phase: level, level control, consistency, and consistency control. Each female was tested for her mating preference four times, once in each treatment (resulting in $N=192$ mate choice trials, randomised testing order, $48 \mathrm{~h}$ between consecutive trials). A difference in female preference between trials with the presence of a virtual opponent (level and consistency treatment) vs. absence of such opponent (level control and consistency control treatment) would validate that female preference is related to a male's distance to an opponent (i.e. apparent aggression) and not simply to male spatial position per se. However, such an effect was expected only if there was an effect of male apparent aggression on female mating preference in trials with a virtual opponent present during the observation phase. As this was not the case (see "Results"), an analysis of the control trials would be redundant and not informative. For completeness, we nonetheless present an analysis of the results for control trials in Online Resource 1.

Immediately following the observation phase, the paired stimulus males and the observer female were transferred to a dichotomous mate-choice arena with the female in a central compartment (Fig. 1c) to test for the female's mating preference (e.g. Thünken et al. 2007; Dechaume-Moncharmont et al. 2011). After a 10-min acclimatisation period, the female was allowed to choose between the two stimulus males during a 22-min mate choice phase that was divided into two recording periods of $11 \mathrm{~min}$ each. In between these two recording periods, we switched the two stimulus males in their position (followed by another $5 \mathrm{~min}$ of acclimatisation) to control for any potential female side bias (e.g. Poschadel et al. 2009; Scherer et al. 2017b). To control for male activity, males were kept in clear Plexiglas cylinders (diameter $=8.0 \mathrm{~cm}$ ), located in the middle of their compartments throughout mate choice trials. During recordings, the compartments of the mate choice arena were physically separated (clear Plexiglas), and they were additionally visually separated from each other during the acclimatisation period.

Using Ethovision XT 11, we quantified female association time (time spent within $10 \mathrm{~cm}$ of either male compartment, hereafter preference zone; Fig. 1c) for the two males over both recording periods (videos were analysed for $10 \mathrm{~min}$, no tracking of the first minute) as a proxy for her mating preference (Thünken et al. 2007; Jeswiet and Godin 2011). Female preference for a particular male was calculated as her total association time with that male divided by her total association time spent with both males (e.g. Schlüter et al. 1998; Schlupp et al. 1999; Poschadel et al. 2009). Females that showed an obvious side bias (i.e. spent $>80 \%$ of total association time in a particular preference zone over both recording periods) were excluded from statistical analyses (excluded trials: $N_{\text {level }}=15$, $N_{\text {consistency }}=5, N_{\text {level control }}=8$, and $N_{\text {consistency control }}=8$; resulting in $N_{\text {level }}=33, N_{\text {consistency }}=43, N_{\text {level control }}=40$, and $N_{\text {consistency control }}=40$ remaining trials for analyses). The removal of side-biased females is common practise in mate choice studies (e.g. Schlüter et al. 1998; Schlupp et al. 1999; Dosen and Montgomerie 2004; Hoysak and Godin 2007; Poschadel et al. 2009; Williams and Mendelson 2010; Kniel et al. 2015; Scherer et al. 2018a, b) and is important to control for females that either did not show interest in stimulus males, were frightened, and/or remained motionless in one corner of the experimental tank (Scherer et al. 2016, 2017b). The relatively high threshold of $80 \%$ was chosen to ensure that only females showing a strong side preference were excluded and to be in line with the existing literature (e.g. Hoysak and Godin 2007; Scherer et al. 2018a, b). To validate that the $80 \%$ threshold is biological meaningful, we tested whether side-biased females were less active, and thus more anxious, during mate choice trials compared to females not showing a side bias. We fitted a linear mixed-effects model (LMM) with female activity (total distance moved in $\mathrm{cm}$, sum of both test periods) as the dependent variable and female side bias (yes or no) as predictor variable. We included female ID, treatment (level, level control, consistency, and consistency control), and female mate choice trial number as random terms. Side-biased females were significantly less active than females not exhibiting a strong side preference $\left(\chi^{2}{ }_{1}=19.887\right.$, $P<0.0001$, estimate side-biased females $\pm \mathrm{SE}=2176.4 \pm$ $124.9 \mathrm{~cm}$, estimate not side-biased females $\pm \mathrm{SE}=2750.2 \pm$ $159.1 \mathrm{~cm} ; N_{\text {trials }}=192 ; N=48$ trials per treatment).

For each mate choice trial, the focal female was unfamiliar with the stimulus males (i.e. she had not seen them before). Stimulus males were used once in each treatment and not used more than once per day. We matched paired stimulus males for family (i.e. male pairs consisted of brothers), body size (standard length difference $<5 \%$; mean $\pm \mathrm{SE}=0.216 \pm 0.011 \mathrm{~cm}$ ), natural aggression level (male difference in their distance to virtual opponent; mean $\pm \mathrm{SE}=1.42 \pm 0.12 \mathrm{~cm})$ and natural consistency of aggression (male difference in their consistency in distance to virtual opponent; mean \pm $\mathrm{SE}=1.559 \pm 0.138 \mathrm{~cm})$. 


\section{Experiment 2: female choice for male apparent boldness}

In experiment 2 (February-April 2018), we tested for an effect of the apparent level $(N=60$ preference assessments) and apparent consistency ( $N=60$ preference assessments) of male boldness on female preference. Before mate choice trials, we tested all males $(N=71)$ and females $(N=60)$ for their boldness level twice, with $48 \mathrm{~h}( \pm 15 \mathrm{~min})$ elapsed between tests (see "Boldness test"). We tested for repeatability of boldness and for a sex difference in the level and consistency of behaviour (see "Data analyses"). We started mate choice trials three days after the boldness typing was completed.

\section{Boldness test}

Male and female boldness was assessed as activity under simulated predation risk (total distance moved in $\mathrm{cm}$, hereafter: APR) using animated individuals of Parachanna obscura $(N=4$, mean $\pm \mathrm{SE}$ standard length $=19.3 \pm 0.3 \mathrm{~cm})$, a naturally occurring sympatric fish predator of $P$. pulcher (Scherer et al. 2017a, 2017b). Boldness tests and the subsequent calculation of the average level and inconsistency of behaviour were performed as described in the "Aggression test" in "Experiment 1: female choice for male apparent aggression". Here, we used a 6-min test period and tracked individuals for $5 \mathrm{~min}$ (no tracking of the first minute). For all individuals, the boldness tests were carried out using a virtual predator specimen that focal fish had not seen before. Different to the above protocol, individuals were transferred to the test tanks without their housing pot. Further, we here aligned two observer tanks behind the test tanks (Fig. 2a, b). We included observer tanks in order to perform the boldness tests and the observation phase of mate choice trials under the exact same conditions, minimising effects that may interfere with our prediction of male behaviour exhibited during the observation phase of mate choice trials. During mate choice trials, observer tanks allowed test females to view the apparent boldness of stimulus males (see "Mate choice trials"). During the boldness test, we introduced opposite-sex observers, which were not further used in this experiment into the observer tanks. Observer conspicuousness was reduced using reflecting lighting (LED lights; I-SY-TL5P01; Soaiy Stick \& Push Lamp; Shenzhen, China) and black plastic surrounding of the observer tanks (see Fig. 2a, b).

\section{Mate choice trials}

Similar to the above experiment 1 , females could choose between two paired stimulus males after prior observation of apparent male behaviour (Fig. 2). That is, mate choice trials consisted of an observation phase and a subsequent choice phase (both observation and choice were divided into two test periods, see below). During the observation, paired stimulus males were manipulated to appear either shy or bold to an observer female by placing them in tanks of different ambient water temperatures, whilst viewing a virtual fish predator moving on a nearby computer screen (Fig. 2a, b). We used three different temperature treatments: low $\left(21^{\circ} \mathrm{C}\right)$, medium $\left(25^{\circ} \mathrm{C}\right)$ and high $\left(29^{\circ} \mathrm{C}\right)$ (for all treatments: range of $\pm 1{ }^{\circ} \mathrm{C}$ ). We created an apparent difference in male level of APR by keeping one of the paired stimulus males in medium water temperature (apparent moderate APR) and the other one in either low water temperature (apparent low APR) (Fig. 2a) or high water temperature (apparent high APR) (Fig. 2b). To test female preference for apparent consistency of male boldness, we performed a second mate choice trial using the same pair of males ( $48 \mathrm{~h}$ between repeated tests, range of $\pm 15 \mathrm{~min}$ ). During second mate choice trials, the apparent low-level (or high-level) male was now kept in high (or low) water temperature (apparent high APR) making it appear inconsistent, while the male being previously kept in medium water temperature was again concurrently presented in the same (medium) temperature treatment making it appear consistent.

For efficiency of time, we tested two females simultaneously for their mating preferences (Fig. 2). During the observation phase, each of the two females could only view one male at a time, we therefore divided the observation phase into two test periods (6 min each) with the female observer tanks being switched in their position in between the two test periods of an observation phase (Fig. 2a, b). This way, the two females could observe both males (in succession not simultaneously). All fish were allowed to acclimatise for $10 \mathrm{~min}$ before the first observation period and for another 2 min after female tanks were switched. During acclimatisation periods, the female observer tanks, the stimulus male tanks, and the computer monitor were visually separated from each other using removable opaque screens. Different to the above experiment 1 , we did not change male treatments in between the two test periods of an observation phase of a single mate choice trial (Fig. 2a, b) (behavioural consistency was manipulated by performing a second mate choice trial, see above). The order in which males were presented during the observation phase did not affect female preference. To test this, we fitted a LMM to the data with female preference (see below) for the first male as dependent variable and included male ID, female ID, mate choice trial number (first or second), and male treatment temperature as random effects (no fixed effects included, aka null model, see below). There was no bias in female preference towards (or against) the male they saw first (intercept $\pm \mathrm{SE}=0.497 \pm$ $\left.0.000 ; 95 \% \mathrm{CI}=[0.470,0.526] ; N_{\text {trials }}=120\right)$.

Similar to the mating preference test in experiment 1 , the dichotomous choice test (where both stimulus males were presented simultaneously; Fig. 2c) was performed with two test periods of $11 \mathrm{~min}$, with the males being switched in their position between test periods; initial acclimatisation was 


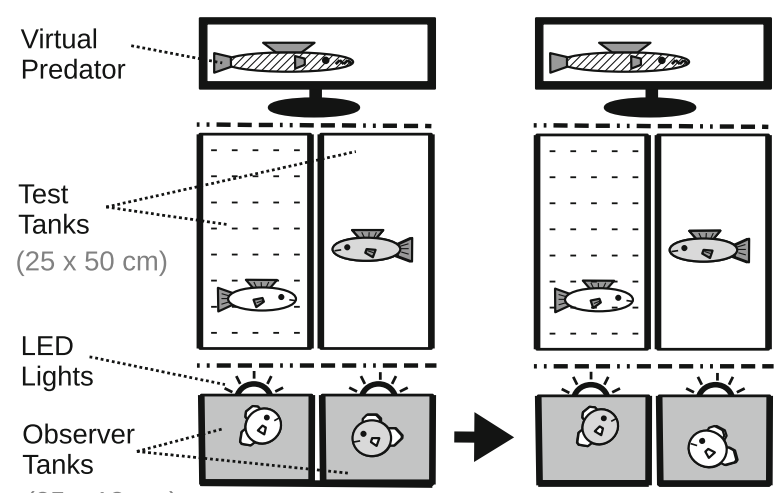

$(25 \times 18 \mathrm{~cm})$ (a)

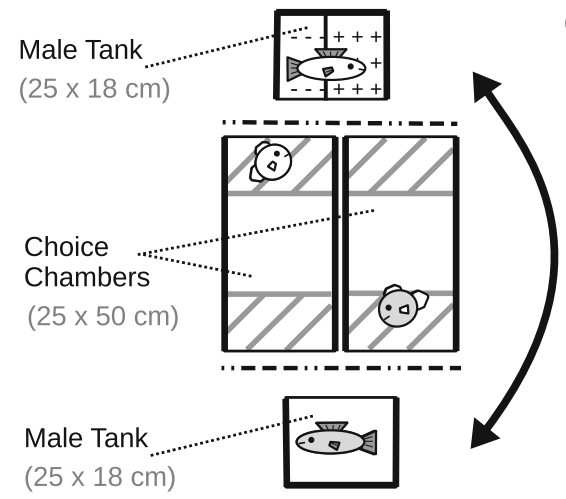

(b)

\section{LEGEND}

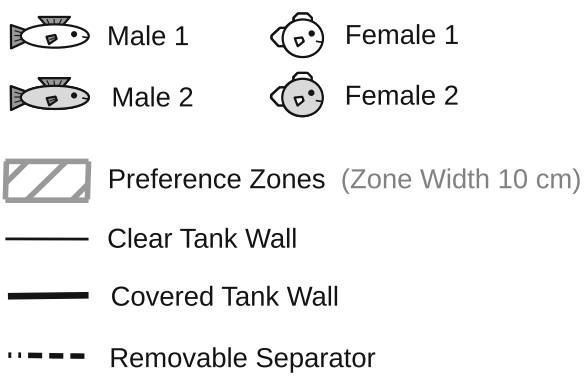

(c)
Fig. 2 Top-view schematic of the experimental set-up for $\mathbf{a}, \mathbf{b}$ the observation and subsequent $\mathbf{c}$ choice phase of mate choice trials in experiment 2. a, b The observation phase set-up was also used for boldness tests. The level and consistency of male boldness was manipulated using low (- - ,

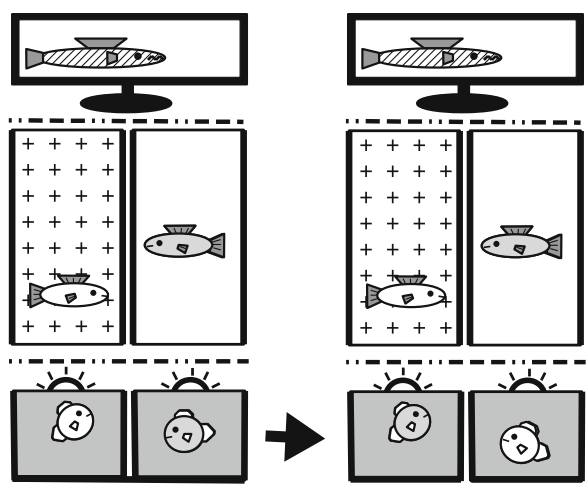

medium (blank tank background) or high $(++)$ water temperature. Arrows indicate that females were switched in their position between the first and second observation periods within a mate choice trial. Grey tank background indicates black tank surrounding (including a black lid)
$10 \mathrm{~min}$, and acclimatisation before the second test period was $5 \mathrm{~min}$. During test periods, the tanks of the mate choice arena were physically separated (clear Plexiglas), whereas they were additionally visually separated using opaque screens during the acclimatisation periods. During the two test periods, males were kept in clear Plexiglas cylinders (diameter = $8 \mathrm{~cm}$ ), positioned in the centre of their respective tanks, ensuring they remained visible to both females throughout the test phase and controlling for male activity. We assessed female preference and female side bias from the association time spent with the two males, as described for experiment 1. Sidebiased females were excluded from preference analyses (excluded trials: $N_{\text {level }}=3, N_{\text {consistency }}=4$ ).

Male treatment temperatures were induced in their individual housing tank 2 days prior to a mate choice trial (using submerged heaters), ensuring sufficient acclimation time $\left(0.17{ }^{\circ} \mathrm{C}\right.$ change/h) to the new temperature regime. Males did not show any signs of distress in response to temperature changes induced. To ensure that temperatures remained constant throughout experimental trials, all experimental tanks were covered externally with polystyrene (apart from tank sides needed to see through; see Fig. 1). The room temperature was set to $20.0{ }^{\circ} \mathrm{C}$ using air conditioning. The water temperature in the female tanks (housing and experimental tanks) was maintained at $25 \pm 1{ }^{\circ} \mathrm{C}$ (equivalent to male medium temperature treatment). In order to avoid an effect of natural male behaviour on mate choice, male pairs were matched as closely as possible for natural inconsistency (mean $\pm \mathrm{SE}$; inconsistency $=193.28 \pm 18.25 \mathrm{~cm}$, within-pair difference in inconsistency $=107.44 \pm 15.99 \mathrm{~cm}$ ) and natural level (mean \pm SE; average APR for all males $=684.21 \pm$ $41.67 \mathrm{~cm}$, within-pair difference of APR $=112.27 \pm$ $18.93 \mathrm{~cm}$ ) in APR. For male pair formation, we did not use the males showing the highest inconsistency values during boldness tests $(N=11)$ in order to efficiently manipulate male behaviour. Therefore, the number of males tested for boldness $\left(N_{\text {males }}=71\right)$ was higher than the number of males used to form male pairs $\left(N_{\text {pairs }}=30, N_{\text {males }}=60\right)$. Males were further matched for standard length as closely as possible (mean \pm SE difference in standard length $=0.11 \pm$ $0.02 \mathrm{~cm}$ ) and for family. 
Our manipulations during the observation phase were effective in manipulating behavioural inconsistency: the inconsistent male showed significantly higher inconsistency than the consistent male in a pair (see Online Resource 2 for statistical analysis and Online Resource 3 for graphical illustration). Further, apparently consistent and inconsistent males did not differ in their apparent level of APR (see Online Resource 2 for statistical analysis and Online Resource 3 for graphical illustration). For our manipulation of the behavioural level, we could confirm that males in the high temperature treatment showed higher APR compared to males in the medium temperature treatment. However, the low and medium temperature treatment males did not differ in their apparent level of APR. Therefore, we restricted the analysis of female preference for the apparent level to mate choice trials where males in the high vs. medium temperature treatment were presented (during the first mate choice trial); that is, all first trials containing low vs. medium temperature treatments were excluded $(N=30$ preference assessments were excluded; resulting in $N=30$ remaining preference assessments). Further, we removed all mate choice trials from the data set where the behavioural manipulation via ambient water temperature was not successful, i.e. in some mate choice trials, the apparently bold male showed a higher level of APR than the apparently shy male (excluded trials: $N=10$ out of 30 trials; $N=20$ remaining trials; total $N$ after removing side biases is $N_{\text {level }}=17$ ) or the apparently consistent male showed higher inconsistency than the apparently inconsistent male (excluded trials: $N=22$ out of 60 trials; $N=38$ remaining trials; total $N$ after removing side biases is $N_{\text {consistency }}=34$ ).

The apparent level of male APR (assessed as outlined in "Boldness test") exhibited during the first test period of the observation phase highly correlated with their apparent level during the second test period of the observation phase (mean \pm SE APR; first test period: $963.346 \pm 30.176 \mathrm{~cm}$; second test period: $875.683 \pm 26.046 \mathrm{~cm}$ ). To test this, we performed an LMM with male APR of the first test period as dependent variable, male APR of the second test period as fixed effect, and male ID as well as mate choice trial number (first or second) as random terms: $\chi^{2}{ }_{1}=56.918, P<0.0001$, intercept $\pm \mathrm{SE}=333.278 \pm 76.314 \mathrm{~cm}$, coefficient $\pm \mathrm{SE}=0.720 \pm$ $0.083 \mathrm{~cm} ; N=120$ test periods of 60 mate choice trials. However, males were significantly more active (exhibited higher APR values) during the first test period of the observation phase compared to the second test period (possibly due to curiosity or excitement). To test this, we performed an LMM with male APR as dependent variable, test period (first or second) as fixed effect, and male ID and mate choice trial number (first or second) as random terms: $\chi_{{ }_{1}}=7.086, P=$ 0.0008 , intercept $\pm \mathrm{SE}=1051.01 \mathrm{~cm}$, coefficient $\pm \mathrm{SE}=$ $-87.66 \pm 32.61 \mathrm{~cm} ; N=240$ test periods of 60 mate choice trials. Due to this behavioural difference, we did not use the average male APR over both test periods but kept these two scores of an observation phase separately for analyses.
We calculated male apparent inconsistency (absolute difference in apparent APR between first and second mate choice trial) from the female's perspective. That is, we calculated two different scores of male apparent inconsistency, one score for each of the two females that saw the male. Each score was based on the very behaviour the female could observe (a female could only observe one male during the first test period and the other one during the second test period of an observation phase). For each male, we thus calculated one score of apparent inconsistency based on the two first observation phase test periods of each mate choice trial and the other score based on the second observation phase test periods (the order in which a female could observe a male was consistent between the two mate choice trials).

\section{Data analyses}

\section{General details}

We performed all data analyses using $\mathrm{R}$ version 3.4.1 ( $\mathrm{R}$ Core Team 2017). If not stated otherwise, LMMs were fitted using the lme 4 package (Bates et al. 2014). Models were simplified using backward model selection, i.e. step-wise removal of the least significant terms. Model assumptions were tested visually using residual and normal q-q plots. For fixed effects, we calculated partial $\mathrm{R}^{2}$ with $\mathrm{CL}$ (confidence level) using the r2glmm package (Jaeger 2016). For insignificant fixed effects, $\mathrm{R}^{2}$ was based on the model before the term was dropped. For all preference analyses, female mating preference was arcsine square-root transformed for normality. Predictor variables were z-transformed for standardisation using the GenABEL package (GenABEL project developers 2013). We checked for collinearity between predictors using Spearman rank correlations (Dormann et al. 2013).

\section{Repeatabilities and sex differences in experiments 1 and 2}

To test for consistent personality differences among individuals, repeatability of aggression and boldness was estimated for males (aggression: $N=96$; boldness: $N=71$ ) and females (aggression: $N=48$; boldness: $N=60$ ) separately by fitting LMMs with ID as random term using the rptR package (Stoffel et al. 2017) (bootstrapping runs $=1000$; permutations $=1000$ ). We further tested for a sex difference in the level of natural aggression and boldness by fitting one LMM to each of the two behaviours (aggression: $N=288$ of 144 individuals; boldness: $N=262$ measurements of 131 individuals; two measurements per individual). We included sex as fixed term and ID as random term in the model. To test for a sex difference in inconsistency, we fitted an LM (linear model) per behaviour to male and female inconsistency (one 
inconsistency measure per individual: aggression: $N=144$; boldness: $N=131$ ) with sex as predictor variable.

\section{Experiment 1: female choice for male apparent aggression}

We tested for a directional female preference for either apparent high-aggression or apparently consistent males by testing for a deviation from random choice (female preference $=0.50$ ) using a null model approach. For each of the two opponent treatments (the level and consistency treatment) separately, we ran a null model with female preference for either the apparent high-aggression male ( $N=33$ mate choice trials) or the apparently consistent male $(N=43$ mate choice trials) as the response variable. As random terms, we included male mate choice trial number and female mate choice trial number. A directional preference would be indicated if the observed mean with $95 \%$ CI (confidence interval) does not include 0.50 .

To test whether male-female behavioural (dis-)similarity had an effect on female preference, we first calculated relative similarity in the level and consistency of aggression between the female and the two paired stimulus males (Scherer et al. 2017b; Scherer and Schuett 2018). We subtracted the similarity (absolute value of the difference in average level or consistency) between the female and the respective high-aggression/consistent male of a pair from the similarity between the female and the low-aggression/inconsistent male. Thus, for positive values of relative similarity, the female's similarity with the high-aggression/consistent male was higher than the female's similarity with the low-aggression/inconsistent male and vice versa. We calculated relative similarity (for level and consistency, respectively) for both male natural behaviour and male apparent behaviour, resulting in four measures of malefemale behavioural similarity; namely, relative similarity in the apparent level of aggression, relative similarity in the apparent consistency of aggression, relative similarity in the natural level of aggression, and relative similarity in the natural consistency of aggression.

For the level treatment, we fitted an LMM on female preference for the apparent high-aggression male $(N=33)$, with relative similarity in the apparent level of aggression as fixed effect. We further included natural level of aggression and relative similarity in natural consistency as covariates. We did not include relative similarity in apparent consistency because this would be the same value for all individuals (as males were standardised for consistency). As random terms, we included male and female mate choice trial number. For the consistency treatment, we fitted an LMM with female preference for apparently consistent males $(N=43)$ as the response variable and included relative similarity in apparent consistency as fixed effect and relative similarity in natural consistency and relative similarity in the natural aggression level as covariates. Again, we used the same random terms as above (male and female mate choice trial number).

We performed all preference analyses also with the data set including side-biased females and present these results in Online Resource 4 (Table 1 for directional preference analyses; Table 2 for (dis-)similarity analyses). Results for preference analyses with and without data from side-biased females are qualitatively similar.

\section{Experiment 2: female choice for male apparent boldness}

We assessed female preference for the level of apparent male boldness using the respective first mate choice trial only. The second mate choice trial was not used to test female preference for the apparent level of behaviour to avoid an interference with previously received information gained during the first mate choice trial. Female preference assessed during the second mate choice test was used to test for an effect of male apparent consistency (difference in apparent APR between the first and second mate choice trial) on female mate choice.

We tested for a directional female preference for the apparently bold or apparently consistent male by fitting a null model (please see "Experiment 1: female choice for male apparent aggression") to the data for each of these two target groups. As random term, we included male pair ID (each pair was used to simultaneously test two females).

To test whether female preference was affected by (dis-)similarity in the apparent level of behaviour, we fitted an LMM on female preference for the apparently bold male. As fixed effects, we included relative similarity in the apparent and natural level of boldness and relative similarity in natural consistency of boldness. Also, we included the difference in the apparent level of aggression between the paired stimulus males as fixed effect to control for the behavioural contrast. We did not include relative similarity in apparent consistency because female preference for the apparent level was assessed prior to the consistency manipulation. As random term, we included male pair ID. For details on the calculation of relative similarities, see "Experiment 1: female choice for male apparent aggression" section. To test for an effect of (dis-)similarity in apparent consistency on female preference, we fitted a similar LMM. The dependent variable was female preference for the apparently consistent male and, as fixed effects, we included relative similarity in apparent consistency, relative similarity in natural consistency, relative similarity in the apparent level (average over the first and second mate choice trial), relative similarity in the natural level of boldness, and male difference in apparent consistency. Again, we included male pair ID as random term.

Similar to the above preference analyses for experiment 1 , we additionally carried out all preference analyses using 
Table 1 Results for sex differences in the level (LMMs) and inconsistency (LMs) of aggression (mean distance to an animated same-sex and same-size opponent) and boldness (activity under simulated predation risk) in experiments 1 and 2. Significant effects highlighted in bold

\begin{tabular}{|c|c|c|c|c|c|c|c|}
\hline Experiment & Trait & $N$ (Trials) & Estimate $\pm \mathrm{SE}(\mathrm{cm})$ & Test statistic & $D F$ & $P$ & $R^{2}[\mathrm{CL}]$ \\
\hline \multirow[t]{2}{*}{1} & Aggression level & 288 & $\begin{array}{l}\text { Males: } 13.49 \pm 1.36 \\
\text { Females: } 15.61 \pm 1.11\end{array}$ & $\chi^{2}=2.415$ & 1 & 0.120 & $0.014[0.000,0.053]$ \\
\hline & Inconsistency of aggression & 144 & $\begin{array}{l}\text { Males: } 4.63 \pm 0.72 \\
\text { Females: } 5.94 \pm 0.59\end{array}$ & $F=3.313$ & 1,142 & 0.071 & $0.023[0.000,0.094]$ \\
\hline \multirow[t]{2}{*}{2} & Boldness level & 262 & $\begin{array}{l}\text { Males: } 682.73 \pm 56.32 \\
\text { Females: } 411.88 \pm 41.47\end{array}$ & $\chi^{2}=21.296$ & 1 & $<0.0001$ & $0.129[0.064,0.210]$ \\
\hline & Inconsistency of boldness & 131 & $\begin{array}{l}\text { Males: } 426.39 \pm 62.52 \\
\text { Females: } 227.55 \pm 33.75\end{array}$ & $F=1.877$ & 1,129 & 0.173 & $0.014[0.000,0.081]$ \\
\hline
\end{tabular}

the data set including side biases. Results are presented in Online Resource 4 (Table 1 for directional preference analyses; Table 3 for (dis-)similarity analyses). The removal of side biases did not change the overall interpretation of the results.

\section{Data availability}

The datasets generated and analysed during the current study are available from the corresponding author on reasonable request.

\section{Results}

\section{Experiment 1: female choice for male apparent aggression}

The natural levels of male and female aggressive behaviour were highly repeatable (males: $\mathrm{R} \pm \mathrm{SE}=0.710 \pm 0.050$, CI [0.598, 0.795], $N_{\text {males }}=96$; females: $\mathrm{R} \pm \mathrm{SE}=0.684 \pm$ 0.078 , CI $[0.511,0.808], N_{\text {females }}=48$ ). Males and females were equally aggressive on average, but males tended to be more consistent in their level of aggression compared to females (Table 1). We found no deviation from random female preference for either the level or consistency of male apparent aggression (Table 2, Fig. 3a). Similarly, we found no effect of relative similarity in the level and consistency of apparent or natural aggression on female preference (Table 3, Fig. 3b). There was a strong trend of female preference decreasing with increasing relative similarity in natural consistency of aggression with the effect size being rather small (Table 3 ).

\section{Experiment 2: female choice for male apparent boldness}

Male and female APR levels were highly repeatable (males: $R \pm \mathrm{SE}=0.637 \pm 0.071$, CI [0.470, 0.752], $N=$ 71; females: $R \pm \mathrm{SE}=0.743 \pm 0.060$, CI [0.605, 0.840], $N=60)$. Males were more active than females when the animated predator was present, though the sexes did not differ in behavioural consistency (Table 1). Females did not show an overall directional preference for the apparent level or consistency of male boldness (Table 2, Fig. 4a). Female preference for the apparently bold male significantly increased with within-male pair difference in the apparent level, such that the larger the difference in male apparent boldness, the greater was the female preference for the bold-appearing male (Table 4, Fig. 5). The difference in male apparent consistency did not affect female preference (Table 4), and there was no effect of relative similarity in apparent/natural boldness (level and consistency) on female preference (Table 4).

Table 2 Results for LMMs testing for a deviation from female random choice for the level and consistency of male apparent aggression (mean distance to an animated same-sex and same-size opponent) and boldness (activity under simulated predation risk) in experiments 1 and 2

\begin{tabular}{|c|c|c|c|c|c|c|}
\hline \multirow[t]{2}{*}{ Experiment } & \multirow[t]{2}{*}{ Response } & \multirow[t]{2}{*}{$N$ (Trials) } & \multicolumn{2}{|c|}{$\begin{array}{l}\text { Female preference arcsine-square root } \\
\text { transformed }\end{array}$} & \multicolumn{2}{|c|}{$\begin{array}{l}\text { Female preference back- } \\
\text { transformed }\end{array}$} \\
\hline & & & Intercept $\pm \mathrm{SE}$ & $95 \% \mathrm{CI}$ & Intercept & $95 \% \mathrm{CI}$ \\
\hline \multirow[t]{2}{*}{1} & Preference apparent high-aggression male & 33 & $0.772 \pm 0.031$ & {$[0.711,0.865]$} & $0.487 \pm 0.001$ & {$[0.415,0.579]$} \\
\hline & Preference apparently consistent male (aggression) & 43 & $0.789 \pm 0.027$ & {$[0.721,0.859]$} & $0.503 \pm 0.001$ & {$[0.435,0.573]$} \\
\hline \multirow[t]{2}{*}{2} & Preference apparently bold male & 17 & $0.771 \pm 0.027$ & {$[0.715,0.826]$} & $0.485 \pm 0.485$ & {$[0.430,0.541]$} \\
\hline & Preference apparently consistent male (boldness) & 34 & $0.784 \pm 0.022$ & {$[0.741,0.827]$} & $0.498 \pm 0.001$ & {$[0.455,0.542]$} \\
\hline
\end{tabular}


Table 3 Results of LMMs testing for an effect of relative similarities (rS) on female preference for male apparent aggression in experiment 1. Female preference data were arcsine square-root transformed for analysis; intercepts and estimates are not back-transformed

\begin{tabular}{|c|c|c|c|c|c|c|c|c|}
\hline Response & Fixed effect & $N$ (Trials) & Intercept $\pm \mathrm{SE}$ & Estimate $\pm \mathrm{SE}$ & $\chi^{2}$ & $D F$ & $P$ & $R^{2}[\mathrm{CL}]$ \\
\hline \multirow{3}{*}{$\begin{array}{l}\text { Preference apparent high } \\
\text { aggression male }\end{array}$} & rS apparent level & \multirow[t]{3}{*}{33} & $0.777 \pm 0.035$ & $0.017 \pm 0.031$ & 0.288 & 1 & 0.591 & $0.009[0.000,0.179]$ \\
\hline & rS natural level & & $0.777 \pm 0.035$ & $-0.006 \pm 0.033$ & 0.028 & 1 & 0.867 & $0.000[0.001,0.151]$ \\
\hline & rS natural consistency & & $0.776 \pm 0.034$ & $0.034 \pm 0.030$ & 1.053 & 1 & 0.305 & $0.036[0.000,0.246]$ \\
\hline \multirow{3}{*}{$\begin{array}{l}\text { Preference apparent consistent } \\
\text { male (aggression) }\end{array}$} & rS apparent consistency & \multirow[t]{3}{*}{43} & $0.788 \pm 0.024$ & $-0.004 \pm 0.024$ & 0.031 & 1 & 0.861 & $0.001[0.000,0.117]$ \\
\hline & rS natural consistency & & $0.788 \pm 0.025$ & $-0.047 \pm 0.023$ & 3.763 & 1 & 0.052 & $0.084[0.001,0.289]$ \\
\hline & rS natural level & & $0.788 \pm 0.024$ & $0.037 \pm 0.023$ & 2.358 & 1 & 0.125 & $0.057[0.000,0.248]$ \\
\hline
\end{tabular}

\section{Discussion}

In the present study, we found no effect of apparent male aggression on female choice. Similarly, apparent male boldness did not affect female choice directly, though female preference for the apparently bold male increased with increasing difference in the apparent boldness level shown by paired stimulus males. Further, there was no effect of natural male aggression/ boldness on female choice in the two experiments. In experiment 1 , female preference tended to decrease with increasing relative similarity in natural consistency of aggression. Males and females showed stable personality variation in both natural aggression and boldness. The sexes were equally aggressive, but males tended to be more consistent in their level of aggression compared to females. Also, males were more active than females (in the presence of the animated predator) but the sexes did not differ in their consistency of boldness.

The absence of a direct effect of apparent behaviour in both experiments may be explained by at least two possible reasons. First, there may not be a causal relationship between the two behavioural traits and female choice. This further implies that the previously found associations of natural male aggression (Scherer et al. 2018a) and boldness (Scherer et al. 2017b) with female mating preference in the rainbow krib were caused by a correlation of the behavioural traits with other traits that are important during mate choice, such as colouration (Godin and Dugatkin 1996; Mafli et al. 2011). However, rainbow kribs provide extensive parental care and both aggression and boldness generally affect the style and amount of care given by parents in many species (reviewed in Chira 2014). In the rainbow krib, territorial breeding pairs guard their fry and protect them from con- and heterospecific intruders for several weeks. Considering that such parental activities are crucial to the reproductive success of (bi)parental species (e.g. Clutton-Brock 1991), it seems likely that there is a causal link between either of the two behavioural traits and mate choice in our model species. However, mate choice is non-trivial and can be cognitive challenging. Instead of choosing a mate for its behaviour, individuals may also derive behavioural benefits from post-pairing behavioural convergence (Laubu et al. 2016).
Second, there may be a causal link between the two behavioural traits and female choice but our behavioural manipulations were not effective in revealing it. That is, in experiment 1 , we found no difference in female preference between the treatment and respective control conditions (for both the apparent level and consistency of aggression). Given our data, we cannot conclusively distinguish whether females did not choose males for their apparent aggression or whether they did not perceive males to be differently aggressive based on our manipulation method. Further, in each of the two experiments presented here, we aimed to manipulate apparent male behaviour by addressing a single variable. In experiment 1 , apparent male aggression was manipulated by fixing a male's average distance to an opponent. In experiment 2 , apparent male boldness was manipulated using a gradient in ambient water temperature. Natural male aggression and boldness could still be expressed via fin postures or body colouration. Fish widely use their fins as a communication channel: aggressiveness is mediated using fin displays (e.g. Riebli et al. 2011) while clamped fins signal anxiety or discomfort (US, personal observation). Rainbow kribs can quickly change their body colouration (US, personal observation). The expression of aggression is usually associated with a strong boost in colourful body pigmentation, but when encountering a potential threat the fish can become very pale or dark in their overall colour appearance (US, personal observation). However, the paired stimulus males used for a mate choice trial were always matched for natural behaviour (and family) as closely as possible. Hence, we would not expect much difference in fin postures or colouration between the two paired stimulus males, which in turn does not leave much room for mate choice based on natural male behaviour. In addition, a discrepancy in apparent and natural male behaviour may provide the female with conflicting information that counterbalance each other, resolving into random choice with regard to male behaviour.

In experiment 2, we found an effect of within male pair contrast in the apparent level of boldness on female preference (the larger the difference the higher female preference for the apparently bold male), supporting the possibility that there is a causal link between boldness and female choice. The direction of effect indicates a general female preference for bolder males, which may have several adaptive advantages. A higher level of 


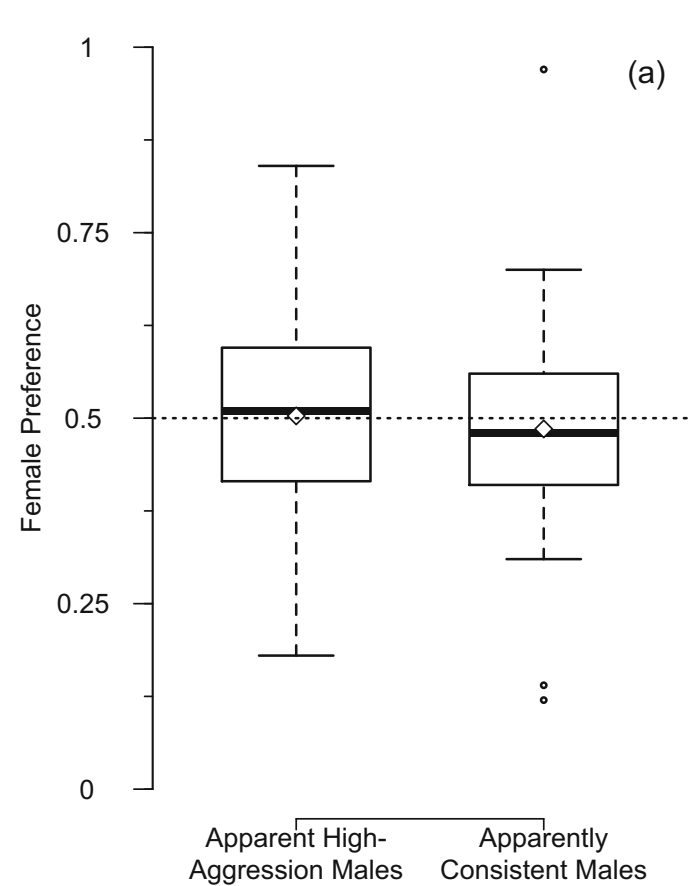

Fig. 3 Female preference in relation to male apparent aggression in experiment 1: a female preference for apparent high-aggression and apparently consistent males, and female preference in relation to relative similarity in $\mathbf{b}$ the apparent level and $\mathbf{c}$ consistency of aggression. a-c
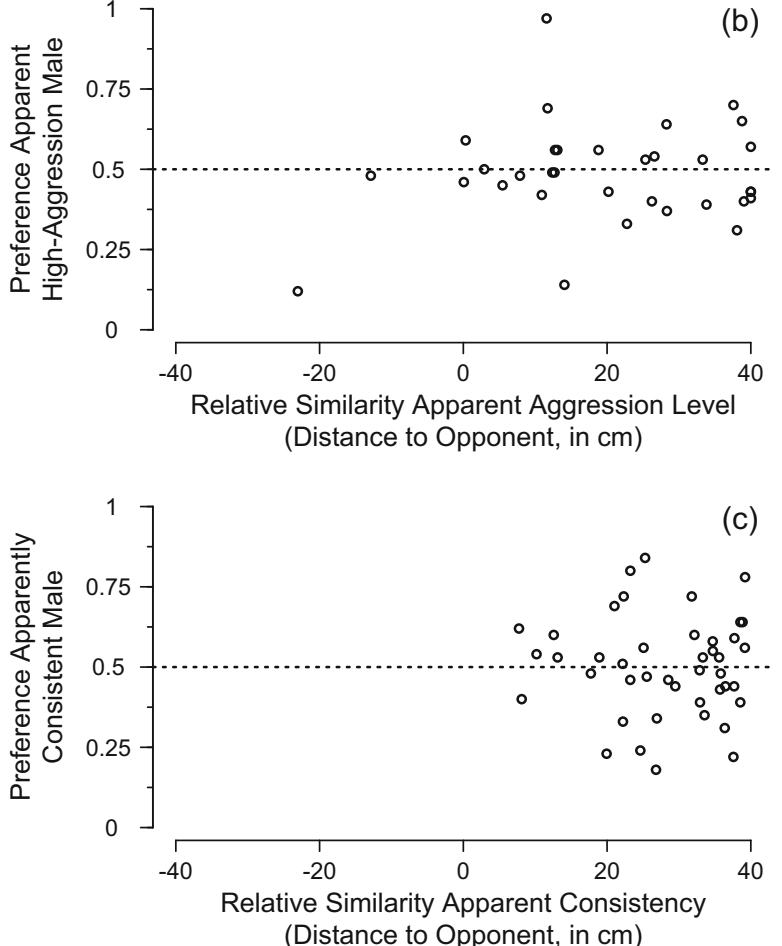

Dotted line indicates random female choice. a The boxes encompass interquartile ranges, whiskers extend with 1.5 interquartile ranges to the most extreme data points, circles represent outliers, medians are represented by thick horizontal lines and means are shown by rhombi

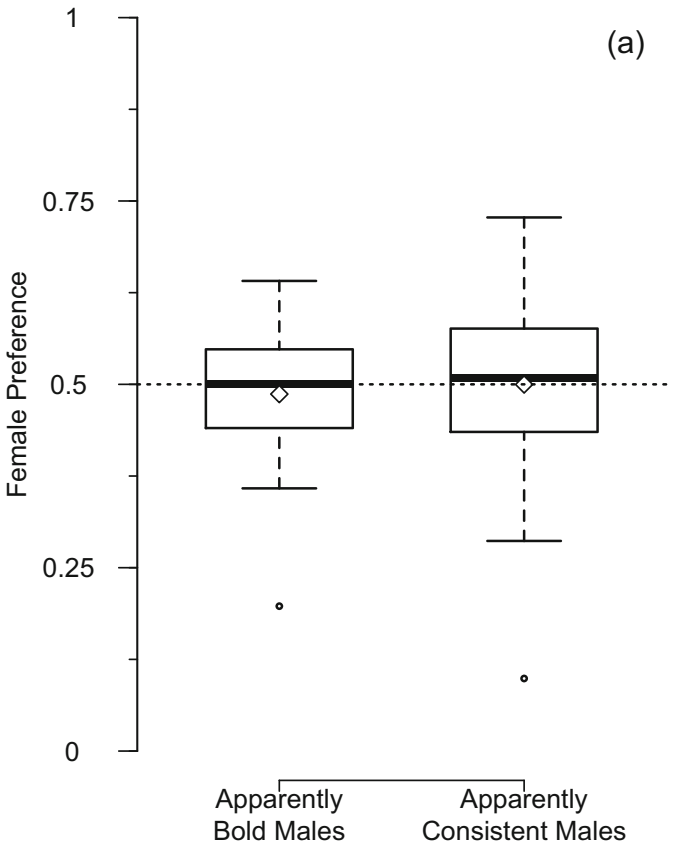

Fig. 4 Female preference in relation to male apparent boldness in experiment 2: a female preference for apparently bold and consistent males, and female preference in relation to relative similarity in $\mathbf{b}$ the apparent level and $\mathbf{c}$ consistency of boldness. a-c Dotted line indicates
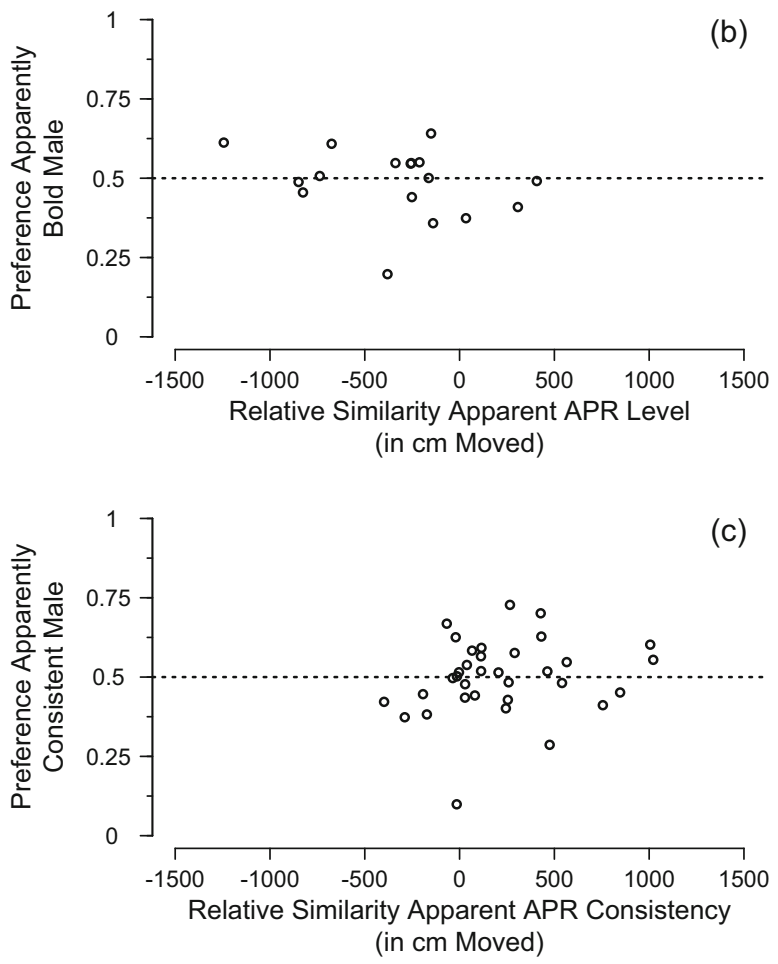

random female choice. a The boxes encompass interquartile ranges, whiskers extend with 1.5 interquartile ranges to the most extreme data points, circles represent outliers, medians are represented by thick horizontal lines and means are shown by rhombi 
Table 4 Results of LMMs testing for an effect of relative similarities (rS) and male behavioural difference on female preferences for male apparent boldness in experiment 2. Female preference was arcsine square-root transformed for analysis, intercepts and estimates are not back-transformed. Significant effects highlighted in bold

\begin{tabular}{|c|c|c|c|c|c|c|c|c|}
\hline Response & Fixed effect & $N$ (Trials) & Intercept $\pm \mathrm{SE}$ & Estimate $\pm \mathrm{SE}$ & $\chi^{2}$ & DF & $P$ & $R^{2}[\mathrm{CL}]$ \\
\hline \multirow{4}{*}{$\begin{array}{l}\text { Preference apparently } \\
\text { bold male }\end{array}$} & rS apparent level & \multirow[t]{4}{*}{17} & $0.771 \pm 0.022$ & $0.009 \pm 0.029$ & 0.101 & 1 & 0.751 & $0.006[0.000,0.296]$ \\
\hline & rS natural level & & $0.771 \pm 0.022$ & $-0.001 \pm 0.024$ & 0.000 & 1 & 0.981 & $0.000[0.000,0.277]$ \\
\hline & Difference apparent level & & $0.771 \pm 0.023$ & $0.061 \pm 0.023$ & 5.638 & 1 & 0.018 & $0.295[0.025,0.638]$ \\
\hline & rS natural consistency & & $0.771 \pm 0.023$ & $0.014 \pm 0.023$ & 0.337 & 1 & 0.562 & $0.021[0.000,0.333]$ \\
\hline \multirow{5}{*}{$\begin{array}{l}\text { Preference apparently } \\
\text { consistent male } \\
\text { (boldness) }\end{array}$} & rS apparent consistency & \multirow[t]{5}{*}{34} & $0.784 \pm 0.021$ & $0.025 \pm 0.022$ & 1.288 & 1 & 0.256 & $0.038[0.000,0.246]$ \\
\hline & rS natural consistency & & $0.784 \pm 0.021$ & $-0.009 \pm 0.023$ & 0.153 & 1 & 0.696 & $0.005[0.000,0.161]$ \\
\hline & Difference apparent consistency & & $0.784 \pm 0.021$ & $-0.042 \pm 0.034$ & 1.481 & 1 & 0.224 & $0.044[0.000,0.256]$ \\
\hline & rS apparent level & & $0.784 \pm 0.021$ & $0.017 \pm 0.023$ & 0.525 & 1 & 0.469 & $0.016[0.000,0.195]$ \\
\hline & rS natural level & & $0.784 \pm 0.021$ & $0.003 \pm 0.023$ & 0.015 & 1 & 0.902 & $0.000[0.000,0.145]$ \\
\hline
\end{tabular}

boldness is associated with increased reproductive success (in the zebrafish, Danio rerio, and in the largemouth bass, Micropterus salmoides; Ariyomo and Watt 2012; Ballew et al. 2017), faster decision making (in three-spined sticklebacks, Gasterosteus aculeatus; Mamuneas et al. 2015), higher foraging success (in guppies, Poecilia reticulata; Dyer et al. 2008), larger body size (Brachyrhaphis episcopi; Brown et al. 2007), and a higher amount of parental provisioning in terms of fin digging (in the convict cichlid, Archocentrus nigrofasciatus; Zworykin and Budaev 2000). However, a directional preference seemingly conflicts with the result of our correlative mate choice study (Scherer et al. 2017b) where we found a dis-assortative female preference for male boldness (i.e. females preferred males of a dis-similar level of boldness). This discrepancy in the direction of effect found may be attributed to the fact that, in the present study, males were generally bolder compared to females. Thus, females were often more similar to the apparently shy male than to the apparently bold male (indicated by negative relative similarity values). In a mate choice trial with such a similarity distribution, a female would be expected to prefer the bold male irrespectively of whether the underlying preference pattern is of dis-assortative

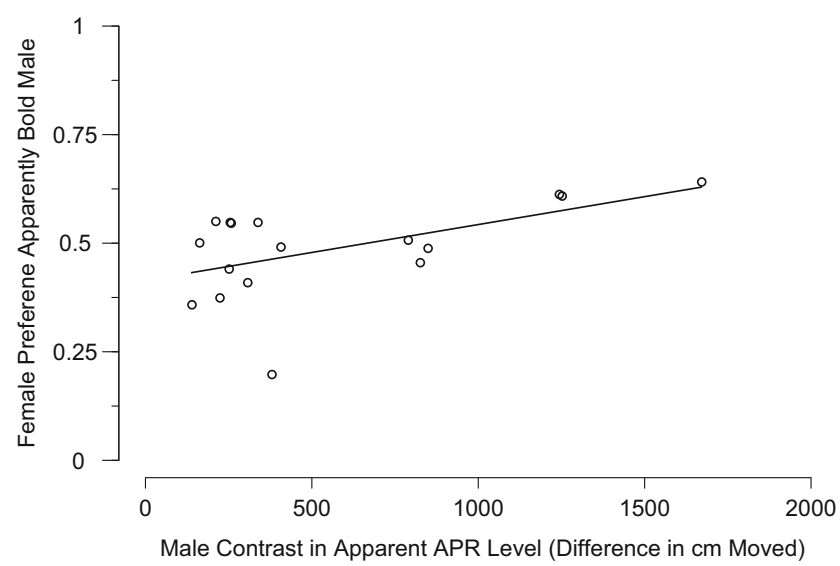

Fig. 5 Female preference in relation to within-male pair difference in the apparent APR level and directional nature. Thus, female preference for bolder males in the present study does not exclude dis-assortment. Possibly, there was not enough variation in relative behavioural similarity to detect dis-assortment (we often obtained negative values for relative similarity in the apparent behavioural level). Our result also indicates that the apparent behavioural contrast created between paired stimulus males was not large enough to provoke a response in female preference (at least for part of the paired stimulus males). As mentioned above, possibly conflicting information between apparent and natural male boldness may necessitate a larger difference in the apparent behaviour in order to overpower other signals.

Notably, natural male aggression/boldness also did not affect female preference, though both traits have been shown to be important in mate choice of female rainbow kribs (Scherer et al. 2017b, 2018a). This is not surprising since we matched paired stimulus males for their natural level and consistency of behaviour. The difference in their natural behaviour was rather low and should not further affect female preference here. However, in experiment 1, male-female similarity in natural consistency tended to affect female preference, which indicates that natural male behaviour could at least partly be sensed by females (e.g. through body colouration or fin postures, see above).

Male and female natural aggressiveness did not differ from each other, which is consistent with the species' biology: both sexes engage into offspring and territory defence. However, males tended to show higher behavioural consistency. In socially monogamous and bi-parental cichlid species, it can often be observed that males do a greater proportion of parental defence behaviours than females (which in turn provide more direct care) (Itzkowitz 1984; Lavery and Reebs 1994; McKaye and Murry 2008). Thus, male aggressiveness might be better conserved than female aggressiveness due to its higher relevance during parental care (reviewed in Schuett et al. 2010). Further, males showed higher activity levels when being exposed to an animated predator compared to females. This behavioural 
difference may be caused by a morphological difference between the sexes: male rainbow kribs are generally larger than females and activity was positively correlated with body size (US et al., unpublished data).

Taken together, we did not find a direct effect of apparent male behaviour on female choice, but our data suggest that the contrast in apparent male behaviour either was not large enough or was not sufficiently distinct from natural male behaviour (at least in experiment 2) to reveal such an effect if one exists. Future studies may follow up on our behavioural manipulation via temperature gradient using a more refined methodology. This would be interesting as we did not exploit the full range of our species' temperature tolerance, especially for mate choice trials testing for an effect of the apparent behavioural level wherein we only used a relatively low between-treatment contrast (which allowed us to manipulate behavioural consistency in subsequent mate choice trials). Furthermore, future directions may involve digital methods that provide a powerful tool to specifically manipulate behaviour. For example, Gierszewski et al. (2018) provide a software to create 3D fish animations where, among others, the swimming pattern of individual fish can be customised. This seems especially worthwhile as digital methods allow a standardised and more convincing manipulation of the apparent behaviour via manipulation of several variables (instead of just a single variable). Finally, we want to emphasise that the usage of behavioural manipulations is essential to answering current hypotheses regarding the evolution of stable personality variation. Although the precise manipulation of specific behavioural traits is not trivial, there are several promising options that are worth exploring.

Acknowledgements The authors thank Milena Markwart for assistance in animal maintenance. Further, the authors thank François-Xavier Dechaume-Moncharmont and an anonymous reviewer for their constructive comments.

Funding information Open Access funding provided by Projekt DEAL. This study was funded by the DFG (Deutsche Forschungsgemeinschaft, grant to WS: SCHU-2927/2-1).

\section{Compliance with ethical standards}

Conflict of interest The authors declare that they have no conflict of interest.

Ethical approval This study was approved by the German "Behörde für Gesundheit und Verbraucherschutz" (permission number 52/16), following the national guideline for the use of animals. Approval from an ethics committee was not required.

Open Access This article is licensed under a Creative Commons Attribution 4.0 International License, which permits use, sharing, adaptation, distribution and reproduction in any medium or format, as long as you give appropriate credit to the original author(s) and the source, provide a link to the Creative Commons licence, and indicate if changes were made. The images or other third party material in this article are included in the article's Creative Commons licence, unless indicated otherwise in a credit line to the material. If material is not included in the article's Creative Commons licence and your intended use is not permitted by statutory regulation or exceeds the permitted use, you will need to obtain permission directly from the copyright holder. To view a copy of this licence, visit http://creativecommons.org/licenses/by/4.0/.

\section{References}

Ariyomo TO, Watt PJ (2012) The effect of variation in boldness and aggressiveness on the reproductive success of zebrafish. Anim Behav 83:41-46

Ariyomo TO, Carter M, Watt PJ (2013) Heritability of boldness and aggressiveness in the zebrafish. Behav Genet 43:161-167

Ballew NG, Mittelbach GG, Scribner KT (2017) Fitness consequences of boldness in juvenile and adult largemouth bass. Am Nat 189:396-406

Bates D, Maechler M, Bolker B, Walker S (2014) lme4: linear mixedeffects models using Eigen and S4, https://cran.r-project.org/web/ packages/lme4/index.html

Brown C, Jones F, Braithwaite VA (2007) Correlation between boldness and body mass in natural populations of the poeciliid Brachyrhaphis episcopi. J Fish Biol 71:1590-1601

Bshary R, Wickler W, Fricke H (2001) Fish cognition: a primate's eye view. Anim Cogn 5:1-13

Cain KE, Ketterson ED (2013) Costs and benefits of competitive traits in females: aggression, maternal care and reproductive success. PLoS One 8:e77816

Castonguay M, Cyr DG (1998) Effects on temperature on spontaneous and thyroxine-stimulated locomotor activity of Atlantic cod. J Fish Biol 53:303-313

Chira A (2014) How does parental personality influence offspring quality in animals? Ann For Res 57:347-362

Clutton-Brock TH (1991) The evolution of parental care. Princeton University Press, Princeton, NJ

Dechaume-Moncharmont FX, Cornuau JH, Keddar I, Ihle M, Motreuil S, Cezilly F (2011) Rapid assessment of female preference for male size predicts subsequent choice of spawning partner in a socially monogamous cichlid fish. C R Biol 334:906-910

Dingemanse NJ, Réale D (2005) Natural selection and animal personality. Behaviour 142:1165-1190

Dormann CF, Elith J, Bacher S et al (2013) Collinearity: a review of methods to deal with it and a simulation study evaluating their performance. Ecography 36:27-46

Dosen LD, Montgomerie R (2004) Female size influences mate preferences of male guppies. Ethology 110:245-255

Doutrelant C, McGregor PK (2000) Eavesdropping and mate choice in female fighting fish. Behaviour 137:1655-1669

Dyer JRG, Croft DP, Morrell LJ, Krause J (2008) Shoal composition determines foraging success in the guppy. Behav Ecol 20:165-171

Forsatkar MN, Nematollahi MA, Biro PA, Beckmann C (2016) Individual boldness traits influenced by temperature in male Siamese fighting fish. Physiol Behav 165:267-272

GenABEL project developers (2013) GenABEL: genome-wide SNP association analysis. $\mathrm{R}$ package version $1.8-0$, https://cran.r-project. org/src/contrib/Archive/GenABEL/

Gierszewski S, Baker D, Müller K, Hütwohl JM, Kuhnert KD, Witte K (2018) Using the FishSim animation toolchain to investigate fish behavior: a case study on mate-choice copying In sailfin mollies. J Vis Exp 141:e58435

Godin J-GJ, Dugatkin LA (1996) Female mating preference for bold males in the guppy, Poecilia reticulata. P Natl Acad Sci USA 93: 10262-10267 
Gosling SD (2001) From mice to men: what can we learn about personality from animal research? Psychol Bull 127:45-86

Hoysak DJ, Godin J-GJ (2007) Repeatability of male mate choice in the mosquitofish, Gambusia holbrooki. Ethology 113:1007-1018

Itzkowitz M (1984) Parental division of labor in a monogomous fish. Behaviour 89:251-260

Jaeger B (2016) R2glmm: computes R squared for mixed (multilevel) models. $\mathrm{R}$ package version 0.1 .1 , https://cran.r-project.org/web/ packages/r2glmm/index.html

Jeswiet SB, Godin J-GJ (2011) Validation of a method for quantifying male mating preferences in the guppy (Poecilia reticulata). Ethology 117:422-429

Kniel N, Durler C, Hecht I, Heinbach V, Zimmermann L, Witte K (2015) Novel mate preference through mate-choice copying in zebra finches: sexes differ. Behav Ecol 26:647-655

Kralj-Fišer S, Sanguino Mostajo GA, Preik O, Pekár S, Schneider JM (2013) Assortative mating by aggressiveness type in orb weaving spiders. Behav Ecol 24:824-831

Laubu C, Dechaume-Moncharmont FX, Motreuil S, Schweitzer C (2016) Mismatched partners that achieve postpairing behavioral similarity improve their reproductive success. Sci Adv 2:e1501013

Laubu C, Schweitzer C, Motreuil S, Louâpre P, Dechaume-Moncharmont F-X (2017) Mate choice based on behavioural type: do convict cichlids prefer similar partners? Anim Behav 126:281-291

Lavery RJ, Reebs SG (1994) Effect of mate removal on current and subsequent parental care in the convict cichlid (Pisces: Cichlidae). Ethology 97:265-277

Lessells CM, Boag PT (1987) Unrepeatable repeatabilities: a common mistake. Auk 104(1):116-121

Mafli A, Wakamatsu K, Roulin A (2011) Melanin-based coloration predicts aggressiveness and boldness in captive eastern Hermann's tortoises. Anim Behav 81:859-863

Mamuneas D, Spence AJ, Manica A, King AJ (2015) Bolder stickleback fish make faster decisions, but they are not less accurate. Behav Ecol 26:91-96

McKaye KR, Murry BA (2008) Sex role differentiation in brood defense by Nicaraguan cichlid fish, Amphilophus xiloanensis. Caribb J Sci 44:13-20

Ophir AG, Galef BG (2003) Female Japanese quail that 'eavesdrop' on fighting males prefer losers to winners. Anim Behav 66:399-407

Patrick SC, Charmantier A, Weimerskirch H (2013) Differences in boldness are repeatable and heritable in a long-lived marine predator. Ecol Evol 3:4291-4299

Pogány Á, Vincze E, Szurovecz Z, Kosztolányi A, Barta Z, Székely T, Riebel K (2018) Personality assortative female mating preferences in a songbird. Behaviour 155:481-503

Poschadel JR, Plath M, Schlupp I (2009) Divergent female mating preference in a clonal fish. Acta Ethol 12:55-60

R Core Team (2017) R: a language and environment for statistical computing. R Foundation for Statistical Computing, Vienna, Austria https://www.R-project.org/

Réale D, Reader SM, Sol D, McDougall PT, Dingemanse NJ (2007) Integrating animal temperament within ecology and evolution. Biol Rev 82:291-318

Riebli T, Avgan B, Bottini AM, Duc C, Taborsky M, Heg D (2011) Behavioural type affects dominance and growth in staged encounters of cooperatively breeding cichlids. Anim Behav 81:313-323

Scherer U, Schuett W (2018) No male mate choice for female boldness in a bi-parental West African cichlid, the rainbow krib (Pelvicachromis pulcher). PeerJ 6:e5373
Scherer U, Buck M, Schuett W (2016) Lateralisation in agonistic encounters: do mirror tests reflect aggressive behaviour? A study on a West African cichlid. J Fish Biol 89:1866-1872

Scherer U, Godin J-GJ, Schuett W (2017a) Validation of 2D-animated pictures as an investigative tool in the behavioural sciences - a case study with a West African cichlid fish, Pelvicachromis pulcher. Ethology 123:560-570

Scherer U, Kuhnhardt M, Schuett W (2017b) Different or alike? Female rainbow kribs choose males of similar consistency and dissimilar level of boldness. Anim Behav 128:117-124

Scherer U, Kuhnhardt M, Schuett W (2018a) Predictability is attractive: female preference for behaviourally consistent males but no preference for the level of male aggression in a bi-parental cichlid. PLoS One 13:e0195766

Scherer U, Tiedemann R, Schlupp I (2018b) Male size, not female preferences influence female reproductive success in a poeciliid fish (Poecilia latipinna) - a combined behavioural/genetic approach. BMC Res Notes 11:364

Schlupp I, Marler C, Ryan MJ (1994) Benefit to male sailfin mollies of mating with heterospecific females. Science 263:373-374

Schlupp I, Waschulewski M, Ryan MJ (1999) Female preferences for naturally-occurring novel male traits. Behaviour 136:519-527

Schlüter A, Parzefall J, Schlupp I (1998) Female preference for symmetrical vertical bars in male sailfin mollies. Anim Behav 56:147-153

Schneider CA, Rasband WS, Eliceiri KW (2012) NIH Image to ImageJ: 25 years of image analysis. Nat Methods 9:671-675

Schuett W, Tregenza T, Dall SRX (2010) Sexual selection and animal personality. Biol Rev 85:217-246

Schuett W, Dall SRX, Royle NJ (2011a) Pairs of zebra finches with similar 'personalities' make better parents. Anim Behav 81:609-618

Schuett W, Godin J-GJ, Dall SRX (2011b) Do female zebra finches, Taeniopygia guttata, choose their mates based on their 'personality'? Ethology 117:908-917

Schuett W, Nava TF, Rahmlow N, Scherer U (2017) Artificial visible implant elastomer (VIE) tags of different colour and symmetry do not influence mate choice in a cichlid. Behaviour 154:387-402

Smith BR, Blumstein DT (2008) Fitness consequences of personality: a meta-analysis. Behav Ecol 19:448-455

Spoon TR, Millam JR, Owings DH (2006) The importance of mate behavioural compatibility in parenting and reproductive success by cockatiels, Nymphicus hollandicus. Anim Behav 71:315-326

Stoffel MA, Nakagawa S, Schielzeth H (2017) rptR: repeatability estimation and variance decomposition by generalized linear mixed-effects models. Methods Ecol Evol 8:1639-1644

Thünken T, Bakker TCM, Baldauf SA, Kullmann H (2007) Active inbreeding in a cichlid fish and its adaptive significance. Curr Biol 17: 225-229

Williams TH, Mendelson TC (2010) Behavioral isolation based on visual signals in a sympatric pair of darter species. Ethology 116:10381049

Witte K, Godin J-GJ (2010) Mate choice copying and mate quality bias: are they different processes? Behav Ecol 21:193-194

Zworykin DD, Budaev SV (2000) Parental brood provisioning as a component of parental care in neotropical cichlid fishes (Perciformes: Cichlidae). J Ichthyol 40:S271-S280

Publisher's note Springer Nature remains neutral with regard to jurisdictional claims in published maps and institutional affiliations. 